\title{
Multicomponent, Efficient Approach for the Synthesis of Pyrazolo[3,4- $d]$ pyrimidine Derivatives Using Glycerol as Green Solvent
}

\author{
Arun Goyal $^{1}$, Harvinder Singh Sohal ${ }^{1}$, Sanjay Kumar ${ }^{2}$, Rajshree Khare ${ }^{1}$ \\ 1 Department of Chemistry, M.M. University, Mullana (Haryana), India \\ 2 Department of Chemistry, M.M. Modi College, Patiala (Punjab), India
}

\begin{abstract}
An environmentally benign, simple and efficient, one pot procedure for the synthesis of pyrazolo[3,4d]pyrimidine derivatives have been developed by the reaction of aldehyde, 5-methyl-2-phenyl-2,4-dihydro-3Hpyrazol-3-one and urea/thiourea in glycerol. The present method affords high yield in short reaction times and devoid of harsh reaction conditions. All the compounds

Keywords: Glycerol, pyrazolo[3,4-d]pyrimidines, aldehydes, 5-methyl-2-phenyl-2,4-dihydro-3H-pyrazol-3-one, urea, thiourea.
\end{abstract}

\section{Introduction:}

The development of green methodologies with the choice of the solvent from renewable resources has gained much interest in recent years [1]. In this concern, use of water has attracted much attention [2] but water based processes are still subject to limitations due to solubility problems of highly hydrophobic substrates. On the other hand, excellent solvent properties like low toxicity $\left(\mathrm{LD}_{50}\right.$ (oral rat) $12600 \mathrm{mg} / \mathrm{kg}$ ), biodegradability, non-flammability, long liquid range (boiling point $290^{\circ} \mathrm{C}$ ), low vapour pressure and solubility of polar organic compounds made the glycerol an excellent option to use as solvent for organic synthesis [3]. Further with the present emphasis and increasing demand of biodiesel, which is responsible for the excess production of glycerol as side product, triggered the discovery of processes that use glycerol for the synthesis of value added chemicals, as reaction medium and for other applications [4-5]. Recently glycerol has been used for Heck and Suzuki coupling [6], Michael addition [7], Fridel-Crafts type addition, epoxide ring opening [8], synthesis of xanthenes [9] and very recently for the production of benzodiazepines and octahydroacridines [10] etc.

On the second line, pyrazolo[3,4-d]pyrimidine derivatives are an important class of compounds from chemistry as well as pharmacological point of view [11]. These molecules possess a variety of medicinal properties like anti-cancer [12], antimicrobial [13], antiviral [14], anti-inflammatory [15], anti-angiogenic [16], anti-allergic and antileishmanial [17] etc. In addition, pyrazolopyrimidines are a class of sedative and anxiolytic drugs related (in terms of their effect) to benzodiazepines. Further certain pyrazolo[3,4-d]pyrimidine derivatives exhibit phosphodiesterase inhibitory action and other have shown herbicidal activity. The easy and economic availability, excellent features of glycerol and importance of pyrazole fused pyrimidine molecules encouraged us to synthesize pyrazolo[3,4-d]pyrimidine derivatives in glycerol (Scheme 1).

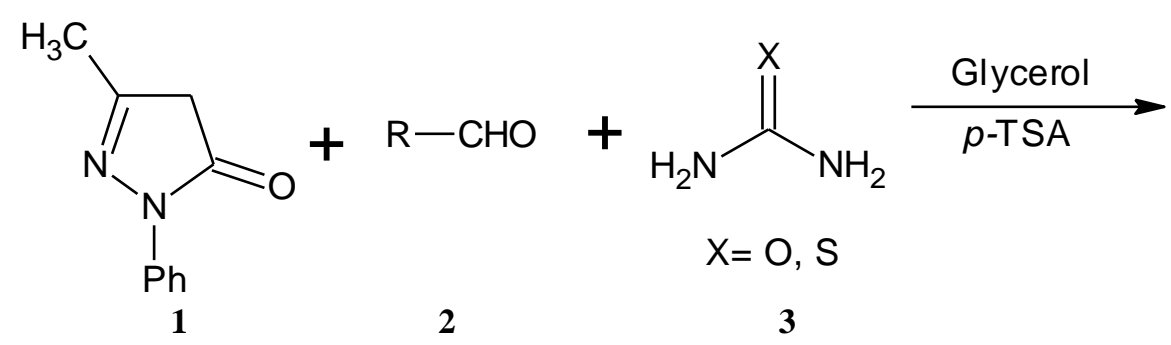

Scheme 1<smiles></smiles>

4a-i, $X=O$

In the pilot experiment, synthesis of 4-(4-chlorophenyl)-3-methyl-1-phenyl-1,3a-dihydro-6H-pyrazolo[3,4$d$ ]pyrimidin-6-one, $\mathbf{4 b}$, has been carried out by the reaction of 5-methyl-2-phenyl-2,4-dihydro-3H-pyrazol-3one, $p$-chlorobenzaldehyde and urea in glycerol in presence of $p$-toulenesulfonic acid ( $p$-TSA). The optimised amount of catalyst found to be $2 \mathrm{~mol} \%$ with respect to aldehyde substrate and afforded the desired product in $91 \%$ yield. Further increase in amount of catalyst did proved fruitful (Table 1, entry 4-6) while decrease in amount of catalyst results in decrease in the yield (Table 1, entry 2). When the reaction was carried out without 
catalyst it was found that glycerol can also promote the reaction but yield was low and afforded the $\mathbf{4 b}$ in $37 \%$ yield.

Table 1

Synthesis of 4-(4-chlorophenyl)-3-methyl-1-phenyl-1,3a-dihydro-6H-pyrazolo[3,4- $d$ ]pyrimidin-6-one, 4b, with varying amount of catalyst

\begin{tabular}{|c|c|c|c|}
\hline S. No. & $\begin{array}{c}\text { Amount of Catalyst } \\
(\mathbf{m o l} \text { \%) }\end{array}$ & Time (hrs) & Yield \\
\hline 1 & 0.0 & 3.8 & $37 \%$ \\
\hline 2 & 1.0 & 2.8 & $77 \%$ \\
\hline 3 & 2.0 & 2.2 & $91 \%$ \\
\hline 4 & 3.0 & 2.2 & $92 \%$ \\
\hline 5 & 4.0 & 2.2 & $92 \%$ \\
\hline 6 & 5.0 & 2.0 & $92 \%$ \\
\hline
\end{tabular}

The structure of compound $\mathbf{4 b}$ was confirmed by the spectral techniques. In IR spectrum absorption at $3424 \mathrm{~cm}^{-}$ ${ }^{1}$ represents the N-H stretching, while absorption at 3074 and $2920 \mathrm{~cm}^{-1}$ represents the aromatic C-H stretching and $\mathrm{sp}^{3}$ hybridised $\mathrm{C}-\mathrm{H}$ stretching respectively. A strong absorption peak for $\mathrm{C}=\mathrm{O}$ is observed at observed at $1600 \mathrm{~cm}^{-1}$. In ${ }^{1} \mathrm{H}$ NMR spectra peaks for nine aromatic protons are observed at $\delta 7.20-7.90$, peak at 5.02 represent $-\mathrm{CH}$ proton and peak for $\mathrm{CH}_{3}$ observed at 2.38. Spectral data of $\mathbf{4 b}$ fully supports the structure assigned to it. After optimizing the conditions, we next examined the generality of this procedure to other substrates using 5-methyl-2-phenyl-2,4-dihydro-3H-pyrazol-3-one, different aromatic aldehydes and urea/thiourea using the similar procedure. The results are summarised in Table 2. Aromatic

Table 2

Synthesis of pyrazolo[3,4-d]pyrimidin-6-one/thione derivatives

\begin{tabular}{|c|l|c|c|c|c|}
\hline S. No. & $\mathrm{R}$ & $\mathrm{X}$ & Time $(\mathrm{hr})$ & Yield & Melting Point $\left({ }^{\circ} \mathrm{C}\right)$ \\
\hline $4 \mathrm{a}$ & $\mathrm{C}_{6} \mathrm{H}_{5}$ & $\mathrm{O}$ & 2.5 & 89 & $182-83$ \\
\hline $4 \mathrm{~b}$ & $4-\mathrm{Cl}_{6} \mathrm{H}_{5}$ & $\mathrm{O}$ & 2.2 & 91 & $213-14$ \\
\hline $4 \mathrm{c}$ & $4-\mathrm{NO}_{2} \mathrm{C}_{6} \mathrm{H}_{5}$ & $\mathrm{O}$ & 2.0 & 90 & $182-84$ \\
\hline $4 \mathrm{~d}$ & $4-\mathrm{OHC}_{6} \mathrm{H}_{5}$ & $\mathrm{O}$ & 2.8 & 88 & $262-63$ \\
\hline $4 \mathrm{e}$ & $4-\mathrm{OMeC}_{6} \mathrm{H}_{5}$ & $\mathrm{O}$ & 3.0 & 85 & $141-42$ \\
\hline $4 \mathrm{f}$ & $3,4-\mathrm{OMeC}_{6} \mathrm{H}_{4}$ & $\mathrm{O}$ & 3.2 & 80 & $158-60$ \\
\hline $4 \mathrm{~g}$ & $3-\mathrm{NO}_{2} \mathrm{C}_{6} \mathrm{H}_{5}$ & $\mathrm{O}$ & 2.5 & 82 & $253-55$ \\
\hline $4 \mathrm{~h}$ & $2-\mathrm{NO}_{2} \mathrm{C}_{6} \mathrm{H}_{5}$ & $\mathrm{O}$ & 3.5 & 79 & $239-40$ \\
\hline $4 \mathrm{i}$ & $2-\mathrm{Cl}_{6} \mathrm{H}_{5}$ & $\mathrm{O}$ & 3.2 & 76 & $262-64$ \\
\hline $4 \mathrm{j}$ & $\mathrm{C}_{6} \mathrm{H}_{5}$ & $\mathrm{~S}$ & 4.0 & 84 & $234-36$ \\
\hline $4 \mathrm{k}$ & $4-\mathrm{Cl}_{6} \mathrm{H}_{5}$ & $\mathrm{~S}$ & 3.2 & 82 & $224-26$ \\
\hline $4 \mathrm{l}$ & $4-\mathrm{NO}_{2} \mathrm{C}_{6} \mathrm{H}_{5}$ & $\mathrm{~S}$ & 3.5 & 80 & $168-69$ \\
\hline $4 \mathrm{~m}$ & $4-\mathrm{OHC}_{6} \mathrm{H}_{5}$ & $\mathrm{~S}$ & 3.0 & 76 & $258-60$ \\
\hline $4 \mathrm{n}$ & $4-\mathrm{OMeC}_{6} \mathrm{H}_{5}$ & $\mathrm{~S}$ & 4.0 & 77 & $170-72$ \\
\hline $4 \mathrm{o}$ & $3-\mathrm{NO}_{2} \mathrm{C}_{6} \mathrm{H}_{5}$ & $\mathrm{~S}$ & 4.0 & 75 & $276-78$ \\
\hline
\end{tabular}

aldehydes carrying different functional groups were subjected to the reaction and in all cases the desired product were obtained in high yields $(75-91 \%)$ in 2.5-4.0 hrs. The method afforded the use of substrates with the formation of any side products.

In conclusion, glycerol was proved to be an effective environmentally benign solvent. The present protocol provides an easier, facile, practically convenient and environmentally benign route for the synthesis of pyrazolo[3,4-d]pyrimidine derivatives and devoid of any harsh reaction condition and toxic organic solvent.

\section{Experimental Section:}

Materials were obtained from commercial suppliers and were used without further purifications. Melting points were recorded in open end capillaries and are uncorrected. ${ }^{1} \mathrm{H}$ NMR spectra were recorded in $\mathrm{CDCl}_{3}$ solution on a Bruker Avance II $400 \mathrm{MHz}$ spectrometer; chemical shifts (delta) are reported in ppm relative to TMS as internal standard. The IR spectra were obtained on a Perkin-Elmer 237B spectrometer.

General Procedure for synthesis of 4-aryl-3-methyl-1-phenyl-1,4,5,7-tetrahydro-6H-pyrazolo[3,4$d]$ pyrimidin-6-one and 4-aryl-3-methyl-1-phenyl-1,4,5,7-tetrahydro-6 $H$-pyrazolo $[3,4-d]$ pyrimidin-6-thione: In a conical flask, aldehyde (1mmole), 3-methyl-1-phenyl-2,4-dihydro-3H-pyrazol-5-one (1mmole), urea $(1.5 \mathrm{mmole}) /$ thiourea $(1.5 \mathrm{mmole}), \quad p$-TSA $(3 \mathrm{~mol} \%)$ and glycerol $5 \mathrm{~mL}$ were taken. Reaction mixture was refluxed for stipulated time as mentioned in table 2. After the completion of reaction, contents are cooled to room temperature and then diluted with ice cold water. Filtered the solid thus obtained and recrystallised with ethanol.

Chracterisation data of the synthesised compounds: 
Multicomponent, Efficient Approach for the Synthesis of Pyrazolo[3,4-d]pyrimidine Derivatives

3-Methyl-1,4-diphenyl-1,4,5,7-tetrahydro-6H-pyrazolo[3,4-d]pyrimidin-6-one, 4a: colourless, $\mathrm{mp}$ : 182 $83^{\circ} \mathrm{C}$; IR $\left(\mathrm{cm}^{-1}\right)$ : 3432, 3057, 2955, 2153, 1811, 1599, 1574, 1409, 1291, 1124, 1099; ${ }^{1} \mathrm{H}$ NMR $\delta$ (ppm): 13.16 $(\mathrm{s}, 1 \mathrm{H}, \mathrm{NH}), 9.97(\mathrm{~s}, 1 \mathrm{H}, \mathrm{NH}), 7.01-7.44(\mathrm{~m}, 10 \mathrm{H}, \mathrm{Ar}-\mathrm{H}), 4.69(\mathrm{~s}, 1 \mathrm{H},-\mathrm{CH}), 2.01\left(\mathrm{~s}, 3 \mathrm{H}, \mathrm{CH}_{3}\right)$; Anal. Calc. for C,H,N (\%): 71.04, 5.30, 18.41; Found C,H,N (\%): 71.02, 5.23, 18.36.

4-(4-Chlorophenyl)-3-methyl-1-phenyl-1,4,5,7-tetrahydro--6H-pyrazolo[3,4-d]pyrimidin-6-one, $\quad 4 \mathrm{~b}$ : colourless solid, mp: $213-14^{\circ} \mathrm{C}$; IR $\left(\mathrm{cm}^{-1}\right): 3424,3074,2920,1600,1570,1448,1188,1124,1004 ;{ }^{1} \mathrm{H}$ NMR $\delta$ (ppm): $13.70(\mathrm{~s}, 1 \mathrm{H}, \mathrm{NH}), 12.29(\mathrm{~s}, 1 \mathrm{H}, \mathrm{NH}), 7.20-7.90(\mathrm{~m}, 9 \mathrm{H}, \mathrm{Ar}-\mathrm{H}), 5.02(\mathrm{~s}, 1 \mathrm{H},-\mathrm{CH}), 2.38\left(\mathrm{~s}, 3 \mathrm{H}, \mathrm{CH}_{3}\right)$, Anal. Calc. for C,H,N (\%): 63.81, 4.46, 16.54; Found C,H,N (\%): 63.72, 4.39, 16.48.

3-Methyl-4-(4-nitrophenyl)-1-phenyl-1,4,5,7-tetrahydro-6 $H$-pyrazolo[3,4- $d]$ pyrimidin-6-one, $\quad 4 \mathrm{c}$ : light yellow, mp: $182-84^{\circ} \mathrm{C}$; IR $\left(\mathrm{cm}^{-1}\right): 3422,3074,2920,2873,2547,2112,1786,1600,1572,1527,1477,1349$, 1194, $1009 ;{ }^{1} \mathrm{H}$ NMR $\delta(\mathrm{ppm}):{ }^{1} \mathrm{H}$ NMR $\delta(\mathrm{ppm}): 13.90(\mathrm{~s}, 1 \mathrm{H}, \mathrm{NH}), 10.57(\mathrm{~s}, 1 \mathrm{H}, \mathrm{NH}), 7.09-8.03(\mathrm{~m}, 9 \mathrm{H}, \mathrm{Ar}-$ $\mathrm{H}), 5.17(\mathrm{~s}, 1 \mathrm{H},-\mathrm{CH}), 2.32\left(\mathrm{~s}, 3 \mathrm{H}, \mathrm{CH}_{3}\right)$, Anal. Calc. for $\mathrm{C}, \mathrm{H}, \mathrm{N}(\%): 61.89,4.33,20.05$; Found $\mathrm{C}, \mathrm{H}, \mathrm{N}(\%)$ : $61.82,4.30,20.01$.

4-(4-Hydroxyphenyl)-3-methyl-1-phenyl-1,4,5,7-tetrahydro-6 $H$-pyrazolo[3,4- $d]$ pyrimidin-6-one, $\quad 4 d$ : Orange solid, mp: $262-63^{\circ} \mathrm{C}$; IR $\left(\mathrm{cm}^{-1}\right): 3178,3086,2870,1654,1558,1543,1458,1411,1328,1289,1150$, 1023; ${ }^{1} \mathrm{H}$ NMR $\delta(\mathrm{ppm}): 10.52(\mathrm{~s}, 1 \mathrm{H}, \mathrm{NH}), 6.90-8.57(\mathrm{~m}, 9 \mathrm{H}, \mathrm{Ar}-\mathrm{H}), 2.34\left(\mathrm{~s}, 3 \mathrm{H}, \mathrm{CH}_{3}\right)$; Anal. Calc. for $\mathrm{C}, \mathrm{H}, \mathrm{N}$ (\%): 67.49, 5.03, 17.49; Found C,H,N (\%): 67.45, 5.00, 17.47.

4-(4-Methoxyphenyl)-3-methyl-1-phenyl-1,4,5,7-tetrahydro-6 $\boldsymbol{H}$-pyrazolo[3,4- $d]$ pyrimidin-6-one, $\quad$ 4e: Orange solid, mp: $141-42^{\circ} \mathrm{C}$; IR $\left(\mathrm{cm}^{-1}\right): 3428,3099,2860,2920,2835,1870,1590,1560,1509,1478,1321$, 1274, 1145, 1011; ${ }^{1} \mathrm{H} \mathrm{NMR}_{\delta}(\mathrm{ppm}): 12.27$ (s, $\left.1 \mathrm{H}, \mathrm{NH}\right), 7.35-8.37$ (m, 9H, Ar-H), 2.30 (s, 3H, $\mathrm{CH}_{3}$ ); Anal. Calc. for C,H,N (\%): 68.25, 5.43, 16.76; Found C,H,N (\%): 68.20, 5.41, 16.70 .

4-(3,4-Dimethoxyphenyl)-3-methyl-1-phenyl-1,4,5,7-tetrahydro-6H-pyrazolo[3,4- $d$ ]pyrimidin-6-one, $\quad 4 f$ : Orange solid, mp: $158-60^{\circ} \mathrm{C}$; IR $\left(\mathrm{cm}^{-1}\right): 3432,3374,3079,2839,1676,1522,1473,1401,1325,1256,1149 ;{ }^{1} \mathrm{H}$ NMR $\delta(\mathrm{ppm})$ : 6.92-7.96 (m, 8H, Ar-H), $4.03(\mathrm{~s}, 1 \mathrm{H},-\mathrm{CH}), 2.36\left(\mathrm{~s}, 3 \mathrm{H}, \mathrm{CH}_{3}\right)$; Anal. Calc. for $\mathrm{C}, \mathrm{H}, \mathrm{N}(\%)$ : 65.92, 5.53, 15.38; Found C,H,N (\%): 65.89, 5.50, 15.37.

3-Methyl-4-(3-nitrophenyl)-1-phenyl-1,4,5,7-tetrahydro-6 $\mathrm{H}$-pyrazolo[3,4-d]pyrimidin-6-one, $\quad 4 \mathrm{g:} \quad$ light orange, mp: $253-55^{\circ} \mathrm{C}$; IR $\left(\mathrm{cm}^{-1}\right): 3372,3267,3086,2937,2845,2710,1599,1535,1487,1360,1225,1134$, $1020 ;{ }^{1} \mathrm{H} \mathrm{NMR}_{\delta}(\mathrm{ppm}):{ }^{1} \mathrm{H}$ NMR $\delta(\mathrm{ppm}): 13.95(\mathrm{~s}, 1 \mathrm{H}, \mathrm{NH}), 10.52(\mathrm{~s}, 1 \mathrm{H}, \mathrm{NH}), 6.95-7.99(\mathrm{~m}, 9 \mathrm{H}, \mathrm{Ar}-\mathrm{H}), 5.18$ (s,1H, -CH), 2.29 (s, 3H, $\mathrm{CH}_{3}$ ), Anal. Calc. for C,H,N (\%): 61.89, 4.33, 20.05; Found C,H,N (\%): 61.84, 4.32, 20.02 .

3-Methyl-4-(2-nitrophenyl)-1-phenyl-1,4,5,7-tetrahydro-6H-pyrazolo[3,4- $d]$ pyrimidin-6-one, $\quad 4$ h: colourless, mp: $239-40^{\circ} \mathrm{C}$; IR $\left(\mathrm{cm}^{-1}\right): 3427,3390,3249,3072,2957,2868,2205,1589,1530,1475,1350,1267$, 1189,$1023 ;{ }^{1} \mathrm{H} \mathrm{NMR}_{\delta}(\mathrm{ppm}):{ }^{1} \mathrm{H}$ NMR $\delta(\mathrm{ppm}): 10.78(\mathrm{~s}, 1 \mathrm{H}, \mathrm{NH}), 7.05-8.15(\mathrm{~m}, 9 \mathrm{H}, \mathrm{Ar}-\mathrm{H}), 5.01(\mathrm{~s}, 1 \mathrm{H},-\mathrm{CH})$, 2.34 (s, 3H, $\mathrm{CH}_{3}$ ), Anal. Calc. for C,H,N (\%): 61.89, 4.33, 20.05; Found C,H,N (\%): 61.85, 4.29, 20.03.

4-(2-Chlorophenyl)-3-methyl-1-phenyl-1,4,5,7-tetrahydro-6H-pyrazolo[3,4- $d]$ pyrimidin-6-one, $\quad 4$ i: colourless solid, mp: $262-64^{\circ} \mathrm{C}$; IR $\left(\mathrm{cm}^{-1}\right)$ : 3397, 3278, 3094, 2899, 2832, 2730, 1610, 1575, 1432, 1379, 1330 , 1249, 1175, 1130, 1025; ${ }^{1} \mathrm{H}$ NMR $\delta(\mathrm{ppm}): 12.16(\mathrm{~s}, 1 \mathrm{H}, \mathrm{NH}), 7.35-8.05$ (m, 9H, Ar-H), $4.95(\mathrm{~s}, 1 \mathrm{H},-\mathrm{CH}), 2.32$ (s, 3H, $\mathrm{CH}_{3}$ ), Anal. Calc. for C,H,N (\%): 63.81, 4.46, 16.54; Found C,H,N (\%): 63.71, 4.42, 16.50 .

3-Methyl-1,4-diphenyl-1,4,5,7-tetrahydro-6H-pyrazolo[3,4-d]pyrimidine-6-thione, $4 \mathbf{j}$ : colourless solid, mp: 234-36 ${ }^{\circ} \mathrm{C}$; IR $\left(\mathrm{cm}^{-1}\right): 3425,3005,1637,1574 ;{ }^{1} \mathrm{H} \mathrm{NMR}_{\delta}(\mathrm{ppm}): 13.87$ (s, 1H, NH), 7.18-7.79 (m, $\left.10 \mathrm{H}, \mathrm{Ar}-\mathrm{H}\right)$, 4.79(s, $1 \mathrm{H},-\mathrm{CH}), 2.39$ (s, 3H, $\mathrm{CH}_{3}$ ); Anal. Calc. for C,H,N (\%): 67.47, 5.03, 17.49; Found C,H,N (\%): 67.45, $5.01,17.44$.

4-(4-Chlorophenyl)-3-methyl-1-phenyl-1,4,5,7-tetrahydro-6 $H$-pyrazolo[3,4- $d]$ pyrimidine-6-thione, $\quad 4 \mathrm{k}$ : colourless, mp: $224-26^{\circ} \mathrm{C}$; IR $\left(\mathrm{cm}^{-1}\right): 3397,3251,3024,2899,2076,1680,1475,1293,1124 ;{ }^{1} \mathrm{H}$ NMR $\delta$ (ppm): $13.71(\mathrm{~s}, 1 \mathrm{H}, \mathrm{NH}), 9.93(\mathrm{~s}, 1 \mathrm{H}, \mathrm{NH}), 7.15-7.86(\mathrm{~m}, 9 \mathrm{H}, \mathrm{Ar}-\mathrm{H}), 4.85(\mathrm{~s}, 1 \mathrm{H},-\mathrm{CH}), 2.31$ (s, 3H, $\left.\mathrm{CH}_{3}\right)$; Anal. Calc. for C,H,N (\%):60.92, 4.26, 15.79; Found C,H,N (\%): 60.73, 4.23, 15.70 .

3-Methyl-4-(4-nitrophenyl)-1-phenyl-1,4,5,7-tetrahydro-6 $\mathrm{H}$-pyrazolo[3,4- $d]$ pyrimidine-6-thione, 4l: light yellow, mp: $168-69^{\circ} \mathrm{C}_{\dot{2}} \mathrm{IR}\left(\mathrm{cm}^{-1}\right): 3397,3240,3167,3060,2932,2830,2573,1610,1580,1487,1350,1188 ;{ }^{1} \mathrm{H}$ $\mathrm{NMR}_{\delta}(\mathrm{ppm}):{ }^{1} \mathrm{H}$ NMR $\delta(\mathrm{ppm}): 13.92(\mathrm{~s}, 1 \mathrm{H}, \mathrm{NH}), 10.48(\mathrm{~s}, 1 \mathrm{H}, \mathrm{NH}), 7.12-8.09(\mathrm{~m}, 9 \mathrm{H}, \mathrm{Ar}-\mathrm{H}), 5.10(\mathrm{~s}, 1 \mathrm{H}$, $\mathrm{CH}$ ), 2.34 (s, 3H, $\mathrm{CH}_{3}$ ), Anal. Calc. for C,H,N (\%): 59.16, 4.14, 19.17; Found C,H,N (\%): 59.14, 4.12, 19.16.

4-(4-Hydroxyphenyl)-3-methyl-1-phenyl-1,4,5,7-tetrahydro-6 $\mathrm{H}$-pyrazolo[3,4-d]pyrimidine-6-thione, $4 \mathrm{~m}$ : orange solid, mp: $258-60^{\circ} \mathrm{C}$; IR $\left(\mathrm{cm}^{-1}\right)$ : 3395, 3325, 3203, 3065, 2926, 1697, 1596, 1533, 1492, 1403, 1204 , 1091; ${ }^{1} \mathrm{H}$ NMR $\delta$ (ppm): 10.51 (s, $\left.1 \mathrm{H}, \mathrm{NH}\right), 6.86-8.53(\mathrm{~m}, 9 \mathrm{H}, \mathrm{Ar}-\mathrm{H}), 2.31$ (s, 3H, $\left.\mathrm{CH}_{3}\right)$; Anal. Calc. for C,H,N (\%): 64.26, 4.79, 16.65; Found C,H,N (\%): 64.24, 4.76, 16.63.

4-(4-Methoxyphenyl)-3-methyl-1-phenyl-1,4,5,7-tetrahydro-6 $\mathrm{H}$-pyrazolo[3,4- $d]$ pyrimidine-6-thione, $\quad 4 \mathrm{n}$ : orange solid, mp: $170-72^{\circ} \mathrm{C}$; IR $\left(\mathrm{cm}^{-1}\right)$ : 3422, 3380, 3205, 3001, 1633, 1573, 1481, 1360, 1273, 1126, 1087; ${ }^{1} \mathrm{H}$ NMR $\delta(\mathrm{ppm}):$ 6.73-8.04 (m, 9H, Ar-H), $2.50\left(\mathrm{~s}, 3 \mathrm{H}, \mathrm{CH}_{3}\right)$; Anal. Calc. for C,H,N (\%): 65.12, 5.18, 15.99; Found C,H,N (\%): 65.03, 5.15, 15.95 . 
3-Methyl-4-(3-nitrophenyl)-1-phenyl-1,4,5,7-tetrahydro-6 $H$-pyrazolo[3,4- $\boldsymbol{d}]$ pyrimidine-6-thione, 4o: light yellow, mp: $276-78^{\circ} \mathrm{C}$; IR $\left(\mathrm{cm}^{-1}\right)$ : 3391, 3247, 3205, 3127, 3028, 2921, 2817, 2572, 1593, 1489, 1376, 1267 , $1189 ;{ }^{1} \mathrm{H} \mathrm{NMR}_{\delta}(\mathrm{ppm}):{ }^{1} \mathrm{H}$ NMR $\delta(\mathrm{ppm}): 12.98(\mathrm{~s}, 1 \mathrm{H}, \mathrm{NH}), 10.43(\mathrm{~s}, 1 \mathrm{H}, \mathrm{NH}), 7.05-8.12(\mathrm{~m}, 9 \mathrm{H}, \mathrm{Ar}-\mathrm{H}), 5.07$ (s,1H, - CH), 2.36 (s, 3H, $\mathrm{CH}_{3}$ ), Anal. Calc. for C,H,N (\%): 59.16, 4.14, 19.17; Found C,H,N (\%): 59.13, 4.11, 19.16.

\section{Acknowledgement:}

The authors thank University Grants Commission, New Delhi, India for the financial support (Sanction No. F.No.8-2 (52)/2011 (MRP/NCRB)).

[1]. (a) S. T. Handy, Chem. Eur. J. 9 (2003) 2938 ;

\section{References}

(b) W. Leitner, Green Chem. 9 (2007) 923

(c) I.T. Horváth, Green Chem. 10 (2008) 1024;

(d) I. Giovanni, H. Silke, L. Dieter, K. Burkhard, Green Chem.8 (2006) 1051;

(e) J.H. Clark, Green Chem. 1 (1999) 1.

[2]. (a) M.-O. Simon, C.-J. Li, Chem. Soc. Rev. 41 (2012) 1415;

(b) R. N. Butler, A. G. Coyne, Chem. Rev. 110 (2010) 6302;

(c) A. Chanda, V. V. Fokin, Chem. Rev. 109 (2009) 725;

(d) C.-J. Li, Chem. Rev. 107 (2007) 2546;

(e) C.-J. Li, Chem. Rev. 105 (2005) 3095;

(f) C.-J. Li, Chem. Rev. 93 (1993) 2023.

[3]. M. Pagliaro, M. Rossi, In The Future of Glycerol: New Usages for a Versatile Raw Material; J. H. Clark, G.A. Kraus, Eds.; RSC Green Chemistry Series: Cambridge, 2008.

[4]. (a) M. Pagliaro, R. Ciriminna, H. Kimura, M. Rossi and C.D. Pina, Angew. Chem., Int. Ed. 46 (2007) 4434;

(b) A. Corma, S. Iborra A. Velty, Chem. Rev. 107 (2007) 2411;

(c) N. Armaroli, V. Balzani, Angew. Chem. Int. Ed. 46 (2007) 52;

(d) F. Jerome, Y. Pouilloux, J. Barrault, ChemSusChem, 1 (2008) 586.

[5]. (a) C.H. Zhou, J.N. Beltramini, Y.X. Fan, G.Q. Lu, Chem. Soc. Rev. 37 (2008) 527;

(b) A. Behr, J. Eilting, K. Irawadi, J. Leschinski, F. Lindner, Green Chem.10 (2008) 13

(c) H. M. Bachhav, S. B. Bhagat, V. N. Telvekar Tetrahedron Lett. 52 (2011) 5697.

(a) A. Wolfson, G. Litvak, C. Dlugy, Y. Shotland, D. Tavor, Ind. Crops Prod. 30 (2009) 78;

(b) A. Wolfson, C. Dlugy, Chem. Pap. 61 (2007) 228;

(c) A. Wolfson, C. Dlugy, Y. Shotland, Environ. Chem. Lett. 5 (2007) 67

[6]. Y. Gu, J. Barrault and F. Jerome, Adv. Synth. Catal. 350 (2008) 2007.

[7]. A. Karam, N. Villandier, M. Delample, C.K. Koerkamp, J.P. Douliez, R. Granet, P. Krausz, J. Barrault and F. Jerome, Chem. Eur. J. 14 (2008) 10196

[8]. F. He, P. Li, Y. Gu, G. Li, Green Chem. 11 (2009) 1767.

(a) C. S. Radatz, R. B. Silva, G. Perin, E. J. Lenardão, R. G. Jacob, D. Alves, Tetrahedron Lett. 52 (2011) 4132;

(b) J.E.R. Nascimento, A. M. Barcellos, M. Sachini, G. Perin, E. J. Lenardão, D. Alves, R. G. Jacob, F. Missau, Tetrahedron Lett. 52 (2011) 2571

[9]. (a) J.Y.L. Brazidec, A. Pasis, B. Tam, C. Boykin, C. Black, D. Wang, G. Claassen, J.H. Chong, J. Chao, J. Fan, K. Nguyen, L. Silvian, L. Ling, L. Zhang, M. Choi, M. Teng, N. Pathan, S. Zhao, T. Li, A. Taveras, Bioorg. Med.Chem. Lett. 22 (2012) 2070; (b) N. Todorovic, E. Awuah, T. Shakya, G.D. Wright, A. Capretta, Tetrahedron Lett. 52 (2011) 5761.

[10]. (a) G.S. Hassan, H.H. Kadry, S.M. Abou-Seri, M.M. Ali, A.E.E.D. Mahmoud, Bioorg. Med. Chem.,19 (2011) 6808;

(b) A.E. Rashad, A.E. Mahmoud, M.M. Ali, Eur. J. Med. Chem. 46 (2011) 1019;(c) M.M. Ghorab, F.A. Ragab, S.I. Alqasoumi, A.M. Alafeefy, S.A. Aboulmagd, Eur. J. Med. Chem. 45 (2010) 171.

[11]. (a) M.A. Gouda, M.A. Berghot, A.I. Shoeib, A.M. Khalil, Eur. J. Med. Chem. 45 (2010) 1843;

(b) M. Bakavoli, G. Bagherzadeh, M. Vaseghifar, A. Shiri, M. Pordel, M. Mashreghi, P. Pordeli, M. Araghi, Eur. J. Med. Chem. 45 (2010) 647;

(c) T.E.S. Ali, Eur. J. Med. Chem. 44 (2009) 4385;

(d) B.S. Holla, M. Mahalinga, M.S. Karthikeyan, P.M. Akberali, N.S. Shetty, Bioorg. Med. Chem. 14 (2006) 2040.

[12]. (a) A.E. Rashad, M.I. Hegab, R.E. Abdel-Megeid, J.A. Micky, F.M.E. Abdel-Megeid, Bioorg. Med. Chem. 16 (2008) 7102;

(b) J.H. Chern, K.S. Shia, T.A. Hsu, C.L. Tai, C.C. Lee, Y.C. Lee, C.S. Chang, S.N. Tseng, S.R. Shih, Bioorg. Med. Chem. Lett. 14 (2004) 2519.

[13]. (a) J.M. Quintela, C. Peinador, L. González, I. Devesa, M.L. Ferrándiz, M.J. Alcaraz, R. Riguera, Bioorg. Med. Chem. 11 (2003) 863;

(b) F. Russo, S. Guccione, G. Romeo, G.U. Barretta, S. Pucci, A. Caruso, M. Amico-Roxas, V. Cutuli, Eur. J. Med. Chem. 28 (1993) 363.

[14]. I. Devesa, M.J. Alcaraz, R. Riguera, M.L. Ferrándiz, Eur. J. Pharmacology 488 (2004) 225

[15]. (a) K. Avasthi, N. Garg, T. Chandra, D.S. Bhakuni, P.P. Gupta, R.C. Srimal, Eur. J. Med. Chem. 28 (1993) 585;

(b) F. Gatta, F. Perotti, L. Gradoni, M. Gramiccia, S. Orsini, G. Palazzo, V. Rossi, Eur. J. Med. Chem. 25 (1990) 419. 